\title{
Breast cancer resistance protein (Bcrp) and the testis—an unexpected turn of events
}

\begin{abstract}
Xiaojing Qian ${ }^{1,2}$, Yan-Ho Cheng ${ }^{3}$, Dolores D Mruk ${ }^{1}$ and C Yan Cheng ${ }^{1}$
Breast cancer resistance protein (Bcrp) is an ATP-dependent efflux drug transporter. It has a diverse spectrum of hydrophilic and hydrophobic substrates ranging from anticancer, antiviral and antihypertensive drugs, to organic anions, antibiotics, phytoestrogens (e.g., genistein, daidzein, coumestrol), xenoestrogens and steroids (e.g., dehydroepiandrosterone sulfate). Bcrp is an integral membrane protein in cancer and normal cells within multiple organs (e.g., brain, placenta, intestine and testis) that maintains cellular homeostasis by extruding drugs and harmful substances from the inside of cells. In the brain, Bcrp is a major component of the bloodbrain barrier located on endothelial cells near tight junctions (TJs). However, Bcrp is absent at the Sertoli cell blood-testis barrier (BTB); instead, it is localized almost exclusively to the endothelial TJ in microvessels in the interstitium and the peritubular myoid cells in the tunica propria. Recent studies have shown that Bcrp is also expressed stage specifically and spatiotemporally by Sertoli and germ cells in the seminiferous epithelium of rat testes, limited only to a testis-specific cell adhesion ultrastructure known as the apical ectoplasmic specialisation (ES) in stage VI-early VIII tubules. These findings suggest that Bcrp is equipped by late spermatids and Sertoli cells to protect late-stage spermatids completing spermiogenesis. Furthermore, Bcrp was found to be associated with $F$ (filamentous)-actin and several actin regulatory proteins at the apical ES and might be involved in the organisation of actin filaments at the apical ES in stage VII-VIII tubules. These findings will be carefully evaluated in this brief review.
\end{abstract}

Asian Journal of Andrology (2013) 15, 455-460; doi:10.1038/aja.2013.24; published online 13 May 2013

Keywords: actin filaments; breast cancer resistant protein; ectoplasmic specialisation; effux drug transporter; germ cells; Sertoli cells; spermatids; spermatogenesis; spermiogenesis; testis

\section{INTRODUCTION}

Breast cancer resistance protein (Bcrp, also known as Abcg2, $\mathrm{Mr}$ $\sim 70 \mathrm{kDa}$ ), as its name implies, was first discovered as an integral membrane protein and a multidrug resistance transporter in adriamycinresistant human MCF-7 (a human breast adenocarcinoma cell line) breast cancer cells approximately 15 years ago. ${ }^{1,2}$ Bcrp is a multidrug resistance ATP-binding cassette (ABC) drug transporter-an efflux drug pump that utilizes ATP in transporting its substrates across the plasma membrane against a steep concentration gradient. Bcrp is a half transporter, as it requires dimerisation of two Bcrp polypeptides (Figure 1) to assemble into a fully functional efflux drug pump. It confers drug resistance by actively pumping chemotherapeutic drugs out of (or preventing their entry into) breast cancer cells, thereby making cancer cells highly resistant to chemotherapy drugs. ${ }^{1,3-5} \mathrm{Bcrp}$, together with P-glycoprotein (Abcb1) and multidrug resistance-associated protein 1 (Mrp1) (Abcc1), constitute the $\mathrm{ABC}$ drug transporter family and since the discovery of P-glycoprotein in ovarian cancer cells some 35 years ago, Bcrp has been a major focus for cancer biologists in developing drug transporter inhibitors. ${ }^{6,7}$ However, after more than three decades of oncological work on $\mathrm{ABC}$ transporters focused on Bcrp, P-glycoprotein and Mrp1, in particular by developing inhibitors against these efflux drug pumps, we are still no closer to confirming the concept that chemotherapy efficacy can be improved by blocking transportermediated drug efflux. ${ }^{8}$ This circumstance, at least in part, is due to the large number of drug transporters ( $>800$ transporters) found in cancer cells. Many of these drug transporters are simultaneously expressed by cancer cells in all types of tumours. Interestingly, these three efflux transporters are not limited to cancer cells. Subsequent studies have shown that they are found in virtually all normal endothelial, epithelial and other cells in multiple organs, including the testes, ${ }^{9-11}$ thus illustrating their roles in regulating normal cellular physiology. Nonetheless, P-glycoprotein and Bcrp are considered to be the two 'gatekeepers' of the blood-tumour barriers that limit the entry of anti-cancer drugs into tumour tissues, such as brain tumours. ${ }^{12}$ While these drug transporters are present in the testes, few studies have examined the testes, and until recently virtually no functional studies, such as how they affect fluid composition in the testes, were found in the literature that examine their role in spermatogenesis. Our interest in studying drug transporters stems from our investigation of developing adjudin, 1-(2,4-dichlorobenzyl)- $1 H$-indazole-3-carbohydrazide, into a male contraceptive. ${ }^{13-15}$ Although this compound is highly potent at disrupting germ cell adhesion in the testes, the drug was shown to have very limited bioavailability following oral administration. ${ }^{16}$ Success in developing this compound into a male contraceptive relies heavily on

${ }^{1}$ The Mary M. Wohlford Laboratory for Male Contraceptive Research, Center for Biomedical Research, Population Council, New York, NY 10065, USA; ${ }^{2}$ Department of Anatomy, Histology and Embryology, School of Basic Medicine, Peking Union Medical College, Beijing 100005, China and ${ }^{3}$ Richmond University Medical Center, Staten Island, NY 10301, USA 


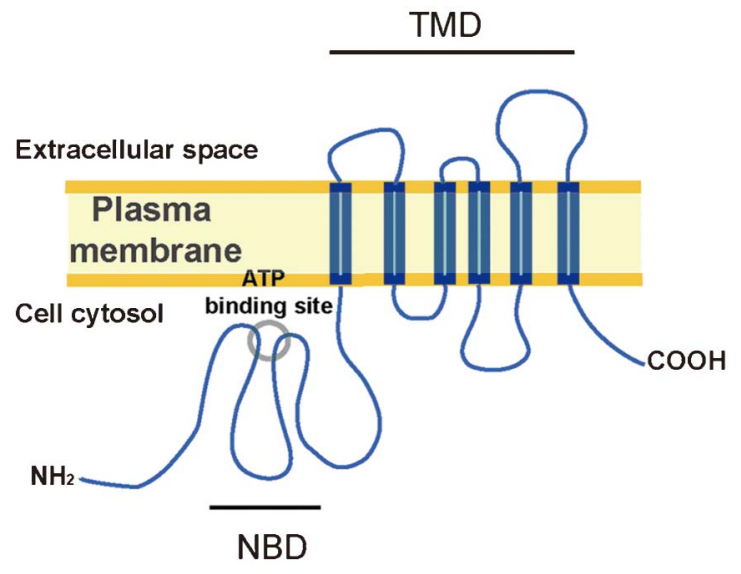

Figure 1 A topographic illustration of different domains of Bcrp. Bcrp is a $\sim 70 \mathrm{kDa}$ polypeptide of 655 amino acids. It has six TMDs, three extracellular domains, four intracellular domains and a NBD near its N-terminus. This drawing depicts only half of a transporter as a homodimerisation of two Bcrp polypeptides is necessary to create a functional Bcrp efflux drug pump, such as in peritubular myoid cells at the tunica propria and also in Sertoli or elongated spermatids at the apical ES. Bcrp, breast cancer resistance protein; ES, ectoplasmic specialisation; NBD, nucleotide binding domain; TMD, transmembrane domain.

our ability to prepare a better formulated version that is both economical to manufacture and that requires a low efficacy dose. ${ }^{17}$ To achieve this goal, we need to better understand the role of drug transporters in determining the bioavailability of adjudin. Indeed, a knockdown of Pglycoprotein by RNAi has been shown to significantly improve the bioavailability of adjudin behind the Sertoli cell blood-testis barrier (BTB) with an increase in influx of $\left[{ }^{3} \mathrm{H}\right]$-adjudin across the Sertoli cell tight junction (TJ)-permeability barrier. ${ }^{18}$ Surprisingly, a knockdown of Pglycoprotein was shown to impede the Sertoli cell TJ-barrier function by rendering the TJ-barrier more 'leaky. ${ }^{18}$ However, while a knockdown of drug influx pumps, such as organic anion transporting polypeptide 3 (Oatp3) (also known as Slcola5, solute carrier organic anion transporter family member 1a5, an influx drug transporter; and a member of the SLC, solute carrier transporter, family), by RNAi also modulated the bioavailability of $\left[{ }^{3} \mathrm{H}\right]$-adjudin to the compartment behind the Sertoli cells, this knockdown, in contrast to P-glycoprotein, did not impede the TJ-barrier function. ${ }^{19}$ Collectively, these findings suggest that the Sertoli cell TJ-barrier function, such as the TJ-permeability barrier, is closely associated with some of the efflux drug pumps, including P-glycoprotein.

In the testes, previous studies have shown that Bcrp is expressed mostly in endothelial cells of microvessels in the interstitium and in peritubular myoid cells in the tunica propria of both humans and mice $e^{3,20,21}$ (Figure 2). Thus, Bcrp is not localized to the BTB in the testes. In this context, it is of interest to note that the BTB is composed of specialized junctions (e.g., basal ectoplasmic specialisation (ES) coexisting with TJs, and gap junctions, as well as desmosomes) $)^{9,22,23}$ between adjacent Sertoli cells that are located near the basement membrane. The BTB also segregates the seminiferous epithelium into the basal and the adluminal compartments (Figure 2). Unlike other blood-tissue barriers, ${ }^{24-26}$ which are composed almost exclusively of capillary endothelial TJs, microvessels in the interstitium between seminiferous epithelium in the testes contribute virtually no barrier function to the BTB. ${ }^{27-30}$ However, in rodents, the peritubular myoid cell layer in the tunica propria that lies behind the basement membrane and the type I collagen layer was found to restrict the diffusion of lanthanum salt and colloidal carbon or thorium in $\sim 75 \%$ of the tubules examined, ${ }^{30,31}$ making it a contributing component to the
BTB. However, myoid cells are less effective at restricting diffusion of substances across the tunica propria in primates, ${ }^{32}$ and perhaps humans. Thus, the BTB is contributed exclusively by Sertoli cells in the testes in primates, and most likely humans.

A recent study, however, showed that Bcrp, in addition to its predominant expression in the endothelial cells of microvessels in the interstitial space and peritubular myoid cells, is also expressed by Sertoli and germ cells, but restricted to the Sertoli/spermatid interface in the seminiferous epithelium known as apical ES (a testis-specific atypical adherens junction). ${ }^{14,25}$ Bcrp displays a restrictive spatiotemporal pattern of expression during the epithelial cycle, such that it is only detected at the apical ES in stage VI-VIII tubules, but not at stage IX-XIV or I-V (Figure 3). ${ }^{33}$ In short, unlike other drug transporters, such as P-glycoprotein and Oatp $3,{ }^{34,35} \mathrm{Bcrp}$ is not a component of the Sertoli cell BTB, ${ }^{33}$ but is stage specifically expressed by Sertoli cells and spermatid in addition to abundant expression by endothelial cells and peritubular myoid cells in rat testes. In this short review, we critically evaluate the likely role of Bcrp in the testes during spermatogenesis in light of these recent findings.

\section{BCrP-STRUCTURE AND FUNCTION}

\section{Structure}

The membrane topology of Bcrp shown in Figure 1 illustrates that the 655 -amino acid polypeptide $(\mathrm{Mr} \sim 70 \mathrm{kDa}$ ) is composed of six transmembrane domains, three extracellular domains, four intracellular domains and a nucleotide binding domain near its $\mathrm{N}$-terminus. ${ }^{36}$ However, this is only a half transporter, and a homodimerisation is necessary to create a functional Bcrp efflux pump.

\section{Substrates}

Bcrp has a wide spectrum of substrates including antibiotics, antiviral drugs, chemotherapeutic drugs (e.g., kinase inhibitors), dietary carcinogens (e.g., heterocyclic amines), HMG-CoA (or 3-hydroxy-3methyl-glutaryl-CoA) reductase inhibitors (drugs that block the enzyme HMG-CoA reductase which plays a central role in producing cholesterol in the liver), endogenous substances (e.g., steroids) and phytoestrogens (Table 1), illustrating that Bcrp can actively pump these drugs/substances out of normal mammalian and cancer cells. In short, the list of $>100$ substrates of Bcrp is growing. It is known that Bcrp has the ability to transport the chemotherapeutic drug gefitinib, an inhibitor of EGFR tyrosine kinase, out of cells, and that multiple protein kinase inhibitors are substrates of Bcrp. ${ }^{37}$ Furthermore, inhibition of phosphoinositide 3 kinase by wortmannin was found to induce translocation of Bcrp from the plasma membrane to cell cytosol, concomitant with a blockade of its transport activity in gall bladder cells. ${ }^{38}$ Additionally, Bcrp interacts and co-localizes with PimIL, a Ser/Thr protein kinase. ${ }^{39}$ Collectively, these findings suggest that Bcrp works closely with protein kinases for phosphorylation and dimerisation, affecting its transport ability and cellular localisation. Furthermore, Bcrp can actively pump dietary carcinogens (Table 1) out of epithelial cells, ${ }^{40}$ which illustrates its protective significance. In addition, the list of phytoestrogens and xenoestrogens that are substrates of Bcrp is growing (Table 1), illustrating its involvement in mediating the steroid microenvironment in the testes via localisation in the myoid cell layer in the testes (Figure 3). In fact, recent studies have shown that steroids, such as oestradiol-17 $\beta$, testosterone and progesterone, can inhibit the transport activity of Bcrp and upregulate Bcrp expression and its cellular localisation in cancer cells, endothelial cells of the brain and endocrine organs. ${ }^{21,41,42}$ Steroids, particularly testosterone and oestrogens, are known to regulate BTB and testicular 


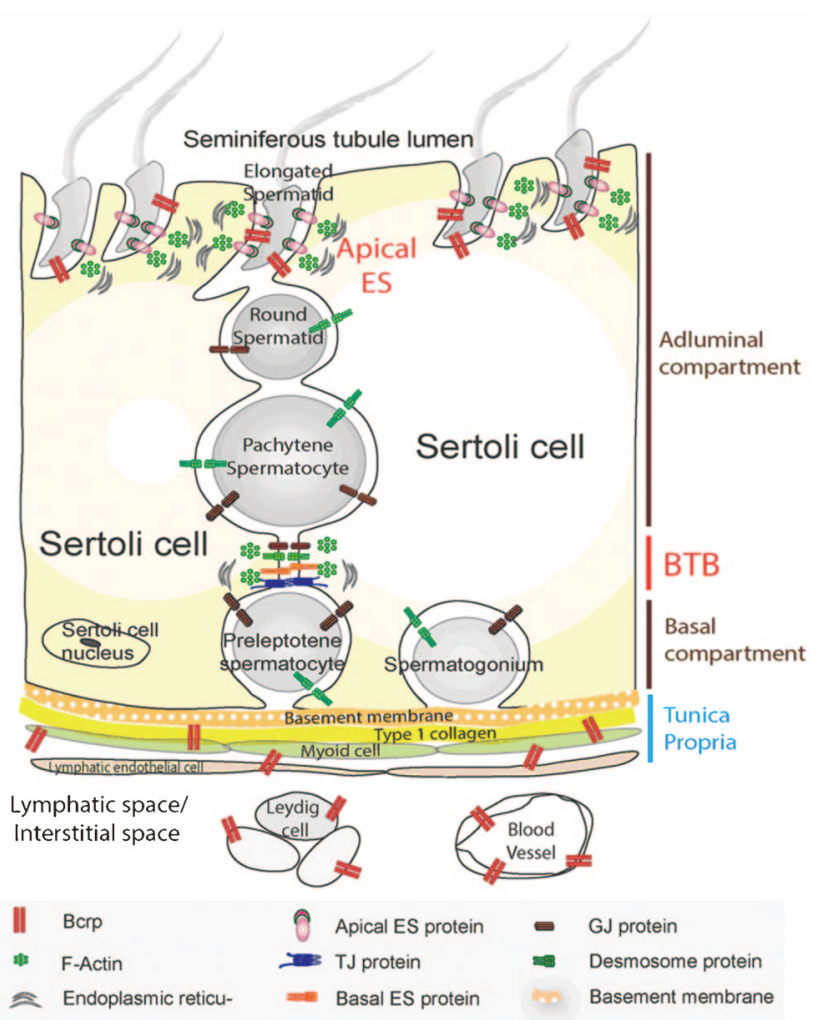

Figure 2 A schematic drawing of the seminiferous epithelium illustrating the spatiotemporal expression of Bcrp in a stage VII-early VIII seminiferous tubule. The BTB anatomically divides the seminiferous epithelium into the basal and the adluminal (apical) compartments. The non-cellular (i.e., basement membrane, type I collagen layer) and cellular (peritubular myoid cells, lymphatic endothelium) zones of the tunica propria are also shown. Different cell junctions in the epithelium between the Sertoli and germ cells are also shown. Bcrp is highly expressed by the endothelial cells of the microvessels in the interstitium and the peritubular myoid cells of the tunica propria behind the basement membrane and the type I collagen layer. However, Bcrp is also expressed by elongated spermatids and Sertoli cells at the apical ES, though it is limited to stage VI-VIII tubules and is predominantly expressed in stage VII tubules. Bcrp, breast cancer resistance protein; BTB, blood-testis barrier; ES, ectoplasmic specialisation; GJ, gap junction; TJ, tight junction.

function, ${ }^{43-46}$ and Bcrp plays a significant role in regulating the transport of steroids across the myoid cells and Sertoli cells at the apical ES (Table 1). Additionally, the activity, expression and cellular distribution of Bcrp are regulated by steroids. ${ }^{21,41,42}$ Collectively, these findings indicate an intimate physiological relationship between Bcrp and the androgen and/or oestrogen microenvironment in the testes, but there are no reports in the literature to assess the role of Bcrp in regulating the level of steroids in the seminiferous epithelium, interstitial fluid and/or the rete testis compartment. However, $\mathrm{Bcrp}^{-1-}$ male mice are fertile, ${ }^{47}$ even though these mice display a malfunction in the disposition of phytoestrogens (e.g., genistein, daidzein, coumestrol) ${ }^{48}$ suggesting that while it is important to testicular function, its loss in function via deletion in male mice can be superseded by other ABC drug transporters, such as P-glycoprotein and Mrp1. Nevertheless, even if the efficacy of spermatogenesis is reduced by $90 \%$ (men produce upwards of 100 million sperm each day after puberty), rodents and men can remain fertile. ${ }^{49}$ Thus, it may be important to re-examine whether there are defects in spermatogenesis in these $\mathrm{Bcrp}^{-1-}$ mice and whether the steroidal contents in the testes of these mice are different from those of wild-type mice.
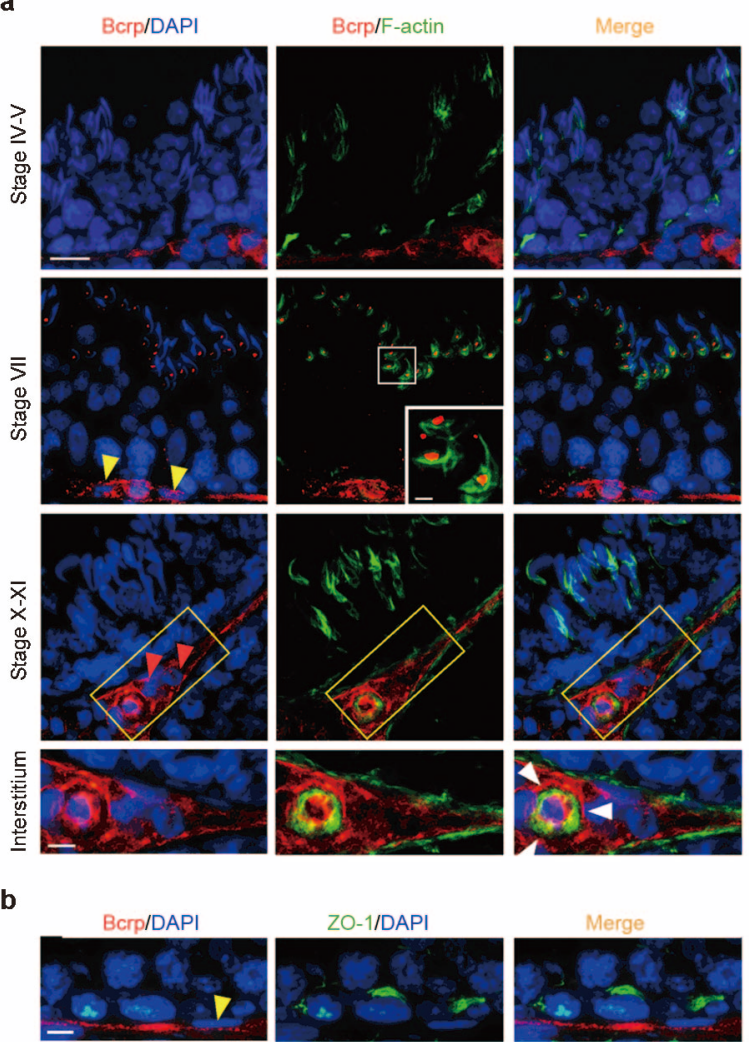

ZO-1/DAPI
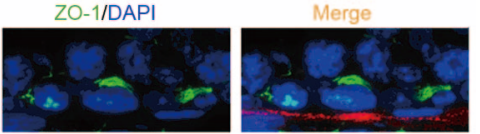

Figure 3 A study by dual-labelled immunofluorescence analysis to examine the cellular localisation and stage-specific expression of Bcrp in the seminiferous epithelium of rat testes. (a) Bcrp, unlike other efflux (e.g., P-glycoprotein $)^{51}$ and influx (e.g., Oatp3) ${ }^{19}$ drug transporters, is not found at the Sertoli cell BTB near the basement membrane as illustrated. Instead, Bcrp (red fluorescence) was detected at the tunica propria associated with peritubular myoid cells (see 'yellow' arrowheads), Leydig cells (see 'red' arrowheads) and most notably with endothelial cells of the microvessels in the interstitium (see 'white' arrowheads annotating a microvessel in which Bcrp (red fluorescence) was found to colocalize with F-actin (green fluorescence) in the microvessel). Interestingly, Bcrp was also detected at the Sertoli/spermatid interface in an ultrastructure known as the apical ES (a testisspecific adherens junction), beginning in late stage $\mathrm{VI}$, most predominantly in stage VII, as shown here, gradually diminishing by stage VIII, and was virtually nondetectable in stage IX. In short, its expression at the apical ES is stage-specific during the epithelial cycle, confined to stages VII-VIII. (b) This experiment clearly illustrates Bcrp is not a component of the Sertoli cell BTB as Bcrp failed to colocalize with the tight junction adaptor protein ZO-1 and other BTB-associated proteins (e.g., N-cadherin). ${ }^{33}$ Dual-labelled immunofluorescence analysis was performed as previously described. ${ }^{33}$ Mouse anti-Bcrp antibody was obtained from Kamiya Biomedical Co. (Seattle, WA, USA; Cat. \# MC-177) and used at a 1:50 dilution. Rabbit anti-ZO-1 was obtained from Invitrogen Life Technologies (Grand Island, NY, USA; Cat. \# 61-7300) and used at a $1: 100$ dilution. Phalloidin-FITC was obtained from Sigma-Aldrich (St Louis, MO, USA; Cat. \# P5282) and used at a $1: 70$ dilution. Scale bar in $\mathbf{a}$ is $50 \mu \mathrm{m}$, or $10 \mu \mathrm{m}$ in the inset and the magnified micrograph of the corresponding boxed area, which applies to other micrographs in $\mathbf{b}$. The scale bar in $\mathbf{b}$ is $10 \mu \mathrm{m}$, which applies to other micrographs in this panel. Bcrp, breast cancer resistance protein; BTB, blood-testis barrier; ES, ectoplasmic specialisation; Oatp3, organic anion transporting polypeptide 3.

\section{LOCALISATION OF Bcrp IN THE TESTIS-AN UNEXPECTED TURN OF EVENTS}

\section{Expression and stage-specific localisation}

In the testes, this integral membrane protein is localized predominantly in the endothelial cells of the microvessels in the interstitial space and in peritubular myoid cells in the tunica propria (Figure 3) 
Table 1 Selected substrates of Bcrp

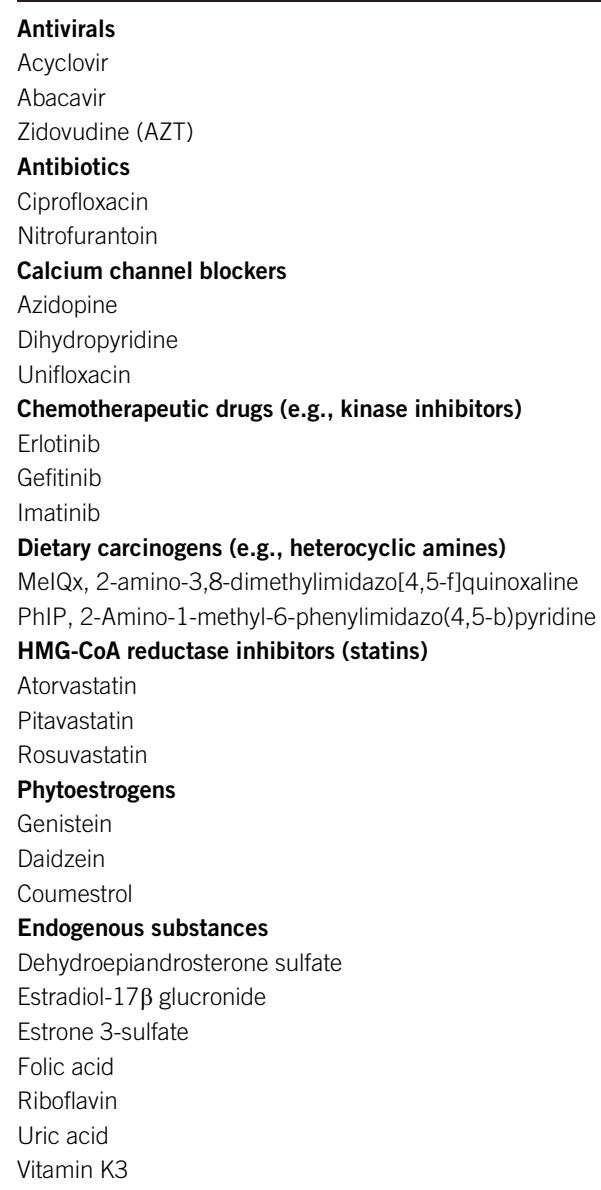

Abbreviation: Bcrp, breast cancer resistance protein.

This list contains selected representatives of substrates of Bcrp based on studies in other mammalian cells including normal mammalian and cancer cells. It is conceivable that Bcrp found in the testis is capable of pumping similar substrates out of the seminiferous epithelium. Detailed information including references can be found in several recent reviews. ${ }^{36,37,62,63}$

as reported previously. ${ }^{20,21,48}$ Unlike P-glycoprotein, which is localized to the BTB and expressed by Sertoli cells, Leydig cells and late spermatids, ${ }^{20,50,51}$ and Mrp1, which is also localized to Sertoli cells at the BTB in humans and mice, ${ }^{20,52}$, but not in endothelial cells in the microvessels of rat testes, ${ }^{53}$ Bcrp is not found at the BTB in rat testes. $^{20,21,33}$ Recent studies have shown that Bcrp is also expressed by Sertoli cells and late spermatids (steps 17-18, but not step 19) in rat testes in a tightly stage-specific pattern at the Sertoli/spermatid interface at the apical ES, in stage VI-VIII tubules (Figure 3). ${ }^{33}$

\section{Bcrp is a component of the apical ES}

A recent study showed that Bcrp structurally interacts with actin and actin-related protein 3 (Arp3), which together with Arp2 form the Arp2/3 complex. This in turn creates a seven-subunit complex with actin-related protein $2 / 3$ complex component 1 to 5 and, when it is activated by neuronal Wiskott-Aldrich syndrome protein, induces branched actin polymerisation that is capable of converting the actin filament bundles at the apical ES from their 'bundled' to a 'de-bundled/ branched' configuration, destabilizing the apical ES function ${ }^{54,55}$ in adult rat testes. ${ }^{33}$ Bcrp also structurally interacts with epidermal growth factor receptor pathway substrate 8 (Eps8), an actin barbed end capping and bundling protein capable of maintaining the actin filament bundles at the apical ES to confer spermatid adhesion and polarity, ${ }^{54,55}$ which illustrates that Bcrp may be involved in F-actin reorganisation at the apical ES. Bcrp is localized mostly to the concave side of the spermatid head in stage VI-VII tubules at the apical ES. It first appears at the apical ES in stage VI tubules, is prominently expressed in stage VII tubules, and its expression rapidly diminishes in stage VIII of the epithelial cycle (Figure 3). ${ }^{33}$ It is noted that the apical ES is an F-actin-rich testis-specific adherens junction, in which bundles of actin filaments that lie perpendicular to the plasma membrane of the Sertoli cell are sandwiched between cisternae of endoplasmic reticulum and the apposing Sertoli-spermatid plasma membrane. ${ }^{14,46,56}$ Once the apical ES appears in step 8 spermatids during spermiogenesis, it is the only anchoring device, replacing desmosomes and gap junctions. It persists until step 19 spermatids when mature spermatozoa are released into the tubule lumen at spermiation. ${ }^{46,56,57}$ Thus, the identification of Bcrp and its association with Eps8 and Arp3 suggest that Bcrp is involved in regulating F-actin organisation, converting actin filaments from their bundled configuration to a branched network, thereby destabilizing the apical ES adhesion function. ${ }^{54,55}$ This phenomenon seems to suggest that Bcrp may be involved in other yet-to-be defined functions during spermatogenesis. Furthermore, the concave side of the spermatid heads in stage VII tubules was recently shown to be the site where extensive endocytic vesicle-mediated protein trafficking occurs, ${ }^{58-60}$ so that 'old' apical ES proteins (e.g., $\beta 1$-integrin, nectin-3, laminin- $\alpha 3,-\beta 3$ and $-\gamma 3$ chains) can be endocytosed and recycled to the newly arisen apical ES in step 8 spermatids during spermiogenesis at stage VIII of the epithelial cycle. ${ }^{46,61}$ Nonetheless, the finding that Bcrp is expressed stage specifically by late spermatids in the adluminal compartment ${ }^{33}$ has shown that this efflux drug pump may be used by late-stage spermatids to safeguard their development by pumping out unwanted substances in the adluminal compartment prior to their transformation to mature spermatozoa. This possibility must be carefully evaluated in future studies.

\section{FUNCTIONAL SIGNIFICANCE OF Bcrp IN SPERMATOGENESIS}

As noted above, unlike other efflux (e.g., P-glycoprotein) ${ }^{18,51}$ and influx (e.g., Oatp3) drug transporters ${ }^{19}$ that are present at the BTB, most notably at the Sertoli/Sertoli cell interface near the basement membrane and colocalized with adhesion protein complexes (e.g., occludin-ZO-1, $\mathrm{N}$-cadherin- $\beta$-catenin, JAM-A-ZO- 1 ) at the BTB, Bcrp is absent at the BTB and does not colocalize with any BTB-associated cell adhesion protein complexes. ${ }^{33}$ Instead, Bcrp is restricted to the endothelium of the microvessels in the interstitial space (Figure 3) and is also detected in peritubular myoid cells in the tunica propria and Leydig cells. ${ }^{33}$ Interestingly, Bcrp is also found at the apical ES at the Sertoli cellelongating/elongated spermatid interface expressed by Sertoli cells and spermatids and is upregulated stage specifically at the apical ES during stage VII. ${ }^{33}$ In short, Bcrp first appears at the apical ES in late stage VI, is highly expressed in stage VII, but is considerably diminished in stage VIII and virtually non-detectable in stage IX (Figure 3), ${ }^{33}$ thus illustrating that this drug efflux transporter is somehow involved in protecting late-stage spermatids (steps 17-19) (or a mechanism being used by late stage spermatids) by sequestering any unwanted toxicants/ drugs from perturbing the final stage of spermiogenesis. More importantly, Bcrp was shown to associate with Eps8, Arp3 and actin at the apical ES, ${ }^{33}$ and a knockdown of Bcrp by RNAi was found to perturb spermatid polarity and adhesion via changes in the localisation of Eps8 and Arp3 at the apical ES in stage VII tubules. These actin regulatory proteins moved away from the apical ES, and their mislocalisation 

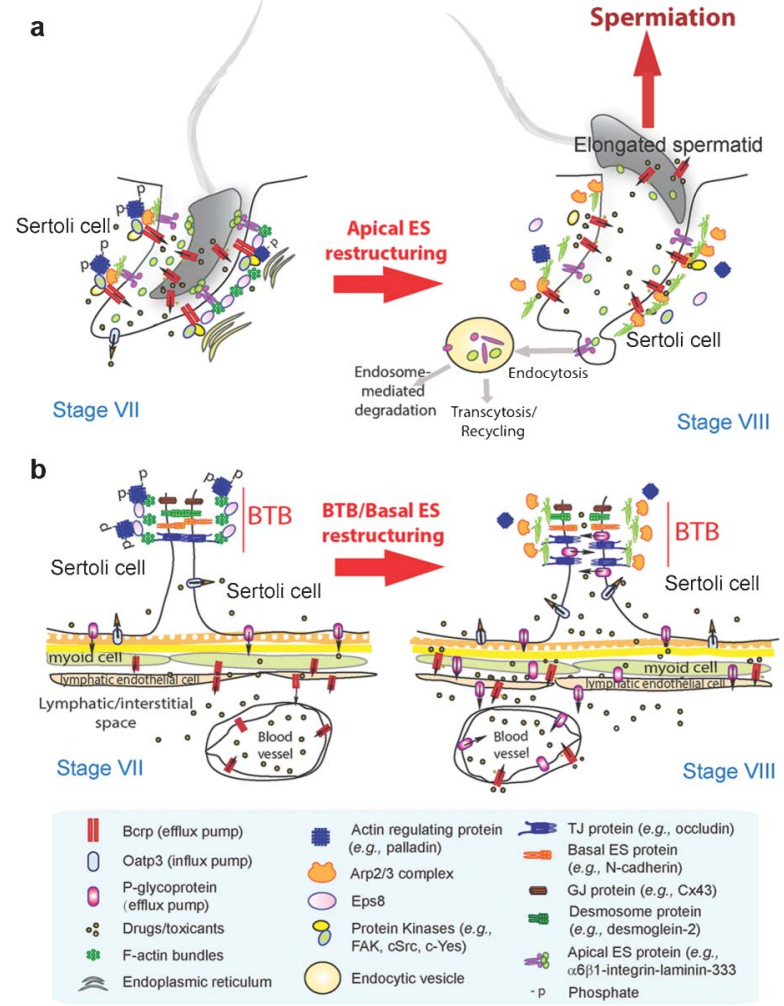

Figure 4 A schematic drawing illustrating the likely function of Bcrp in the seminiferous epithelium of adult rat testes. It is conceivable that Bcrp functions as a drug efflux transporter at all stages of the epithelial cycle and is highly expressed by peritubular myoid cells at the tunica propria and by endothelial cells of the microvessels in the interstitium to prevent the entry of unwanted drugs and/or toxicants that would otherwise perturb spermatogenesis into the seminiferous epithelium. (a) In a stage VII tubule, Bcrp, in addition to serving as an efflux drug transporter to pump harmful substances out of the elongated spermatids (step 19) and Sertoli cells, is highly expressed at the apical ES and may be used to recruit Eps8 to the apical ES to maintain F-actin integrity. Accordingly, actin filament bundles can be properly organized to confer apical ES function given that Bcrp was found to structurally interact with Eps8 and because its knockdown in the testes, in vivo by RNAi, was shown to perturb F-actin organisation at the apical ES in stage VII tubules (see text for details). However, during stage VIII of the cycle, Bcrp may be involved in the recruitment of Arp3 to the apical ES to induce branched actin polymerisation. Thus, actin filament bundles can be 'debundled' and re-organized as a branched actin network. This destabilisation of the apical ES facilitates (i) the release of sperm at spermiation and (ii) endocytic vesicle-mediated protein trafficking so that apical ES proteins can be transcytosed and recycled to assemble 'new' apical ES that arise from spermiogenesis, such as at the interface of step 8 spermatids and Sertoli cells in stage VIII tubules. (b) Bcrp was not found to be expressed by Sertoli cells at the BTB in stage VII-VIII tubules nor in any other stages of the epithelial cycle. However, Bcrp is restricted to the endothelial cells of the microvessels in the interstitium and to the peritubular myoid cells at the tunica propria during both stages to prevent drugs/ toxicants from entering the seminiferous epithelium and perturbing spermatogenesis. As Bcrp is absent at the BTB, Bcrp does not appear to be involved in the restructuring of the F-actin network at the basal ES in stage VIII tubules. Arp3, actin-related protein 3; Bcrp, breast cancer resistance protein; BTB, blood-testis barrier; Eps8, epidermal growth factor receptor pathway substrate 8; ES, ectoplasmic specialisation.

led to changes in the F-actin distribution at the apical ES, thus rendering a disruption of spermatid polarity. ${ }^{33}$ For instance, following the in vivo knockdown of Bcrp by RNAi, F-actin at the apical ES of rats in the treatment $v s$. the control group was considerably reduced, indicating that actin filaments no longer retained their 'bundled' configuration, but 'de-bundled' via a down-regulation and mislocalisation of Eps8. ${ }^{33}$ These findings illustrate that Bcrp may be involved in the organisation of F-actin at the apical ES during spermiogenesis. However, more work is needed to expand this initial observation regarding the precise molecular mechanism(s) by which Bcrp regulates F-actin organisation in the epithelium during the epithelial cycle. Based on these findings, we now provide a hypothetical model regarding the likely function of Bcrp in the seminiferous epithelium of rat testes as shown in Figure 4. It is obvious that this model will be rapidly updated when more data are available from future studies.

\section{CONCLUDING REMARKS AND FUTURE PERSPECTIVES}

In this brief review, we have critically evaluated the likely functional significance of Bcrp in spermatogenesis in light of its unusual cellular distribution and stage-specific expression patterns in the seminiferous epithelium during the epithelial cycle of spermatogenesis. We have also provided a hypothetical model of the likely involvement of Bcrp in regulating apical ES function via its effects on the F-actin network at the ES, mediated by Eps8 and Arp3 in stage VII-VIII tubules, in addition to its normal role of serving as a drug efflux transporter. This hypothesis is supported by functional experiments using RNAi that demonstrated the likely involvement of Bcrp in the organisation of F-actin at the apical ES during spermiogenesis. Further work is warranted to explore the functional significance of this efflux drug transporter in spermatogenesis. For instance, the functional involvement of Bcrp in steps 18-19 spermatids as well as Sertoli cells in stage VI-VIII tubules in excluding drugs, such as adjudin, from the epithelium must be carefully evaluated. This goal may be accomplished by evaluating the ability of staged tubules isolated from rats to transport adjudin across the seminiferous tubule into the tubule lumen.

\section{AUTHOR CONTRIBUTIONS}

CYC conceived the ideas for preparing this review article, wrote the first draft, and revised and prepared the final version of the manuscript. CYC, XQ and YHC performed the literature search, research on Bcrp and other drug transporters and critically evaluated findings in the field. YHC and CYC prepared Table 1. CYC, DDM and XQ critically discussed and evaluated the latest research on the subject of drug transporters, in particular Bcrp. CYC conceived the hypothetical model of Bcrp function at the apical ES. XQ and CYC provided the first draft of the hypothetical model. XQ prepared all the figures. CYC, $\mathrm{XQ}$ and YHC performed the final editing of the manuscript.

\section{COMPETING FINANCIAL INTERESTS}

The authors have nothing to declare.

\section{ACKNOWLEDGMENTS}

Studies from the authors' laboratory were supported by grants from the National Institutes of Health (NICHD, R01 HD056034 to C Yan Cheng; U54 HD029990 Project 5 to C Yan Cheng).

1 Doyle LA, Yang W, Abruzzo LV, Krogmann T, Gao Y et al. A multidrug resistance transporter from human MCF-7 breast cancer cells. Proc Natl Acad Sci USA 1998; 95: 15665-70.

2 Ross DD, Yang W, Abruzzo LV, Dalton WS, Schneider E et al. Atypical multidrug resistance: breast cancer resistance protein messenger RNA expression in mitoxantrone-selected cell lines. J Natl Cacer Inst 1999; 91: 429-33.

3 Natarajan K, Xie Y, Baer MR, Ross DD. Role of breast cancer resistance protein (BCRP/ ABCG2) in cancer drug resistance. Biochem Pharmacol 2012; 83: 1084-103.

4 Nakanishi T, Ross DD. Breast cancer resistance protein (BCRP/ABCG2): its role in multidrug resistance and regulation of its gene expression. Chin J Cancer 2012; 31: 73-99. 
5 Muller F, Fromm MF. Transporter-mediated drug-drug interactions. Pharmacogenomics 2011; 12: 1017-37.

6 Juliano RL, Ling V. A surface glycoprotein modulating drug permeability in Chinese hamster ovary cell mutants. Biochim Biophys Acta 1976; 455: 152-62.

7 Riordan JR, Ling V. Purification of P-glycoprotein from plasma membrane vesicles of Chinese hamster ovary cell mutants with reduced colchicine permeability. J Biol Chem 1979; 254: 12701-5

8 Tamaki A, lerano C, Szakacs G, Robey RW, Bates SE. The controversial role of ABC transporters in clinical oncology. Easays Biochem 2011; 50: 209-32.

9 Cheng $\mathrm{CY}$, Mruk DD. The blood-testis barrier and its implication in male contraception. Pharmacol Rev 2012; 64: 16-64.

10 Kis O, Robillard K, Chan GN, Bendayan R. The complexities of antiretroviral drug-drug interactions: role of ABC and SLC transporters. Trends Pharmacol Sci 2010; 31: 2235.

11 Ashraf $\mathrm{T}$, Kis O, Banerjee N, Bendayan R. Drug transporters at brain barriers: expression and regulation by neurological disorders. Adv Exp Med Biol 2012; 763: 20-69.

12 Agarwal S, Hartz AM, Elmquist WF, Bauer B. Breast cancer resistance protein and Pglycoprotein in brain cancer: two gatekeepers team up. Curr Pharm Des 2011; 17: 2793-802.

13 Mok KW, Mruk DD, Lie PP, Lui WY, Cheng CY. Adjudin, a potential male contraceptive, exerts its effects locally in the seminifeorus epithelium of mammalian testes. Reproduction 2011; 141: 571-80.

14 Mruk DD, Silvestrini B, Cheng CY. Anchoring junctions as drug targets: role in contraceptive development. Pharmacol Rev 2008; 60: 146-80.

15 Cheng CY, Lie PP, Wong EW, Mruk DD, Silvestrini B. Adjudin disrupts spermatogenesis via the action of some unlikely partners: Eps8, Arp2/3 complex, drebrin E, PAR6 and 14-3-3. Spermatogenesis 2011; 1: 291-7.

16 Cheng CY, Mruk DD, Silvestrini B, Bonanomi M, Wong CH et al. AF-2364 [1-(2, 4-dichlorobenzyl)-1H-indazole-3-carbohydrazide] is a potential male contraceptive: a review of recent data. Contraception 2005; 72: 251-61.

17 Mruk DD, Cheng CY. Delivering non-hormonal contraceptives to men: advances and obstacles. Trends Biotechnol 2008; 26: 90-9.

18 Su L, Mruk DD, Lui WY, Lee WM, Cheng CY. P-glycoprotein regulates blood-testis barrier dynamics via its effects on the occludin/zonula occludens 1 (ZO-1) protein complex mediated by focal adhesion kinase (FAK). Proc Natl Acad Sci USA 2011; 108: $19623-8$

19 Su L, Mruk DD, Lee WM, Cheng CY. Drug transporters and blood-testis barrier function. J Endocrinol 2011; 209: 337-51.

20 Bart J, Hollema H, Groen HJ, de Vries EG, Hendrikse NH et al. The distribution of drugefflux pumps, P-gp, BCRP, MRP1 and MRP2, in the normal blood-testis barrier and in primary testicular tumours. Eur J Cancer 2004; 40: 2064-70.

21 Dankers AC, Sweep FC, Pertijs JC, Verweij V, van den Heuvel JJ et al. Localization of breast cancer resistance protein (Bcrp) in endocrine organs and inhibition of its transport activity by steroid hormones. Cell Tissue Res 2012; 349: 551-63.

22 Pelletier RM. The blood-testis barrier: the junctional permeability, the proteins and the lipids. Prog Histochem Cytochem 2011; 46: 49-127.

23 Franca LR, Auharek SA, Hess RA, Dufour JM, Hinton BT. Blood-tissue barriers: morphofunctional and immunological aspects of the blood-testis and bloodepididymal barriers. Adv Exp Med Biol 2012; 763: 237-59.

24 Cheng CY. Biology and Regulation of Blood-Tissus Barriers. Austin, TX: Landes Bioscience/Springer Science+Business Media, LLC; 2012. p1-361.

25 Wong EW, Mruk DD, Cheng CY. Biology and regulation of ectoplasmic specialization, an atypical adherens junction type, in the testis. Biochem Biophys Acta 2008; 1778: 692-708.

26 Wong $\mathrm{CH}$, Cheng $\mathrm{CY}$. The blood-testis barrier: Its biology, regulation and physiological role in spermatogenesis. Curr Topics Dev Biol 2005; 71: 263-96.

27 Setchell BP. Blood-testis barrier, functional and transport proteins and spermatogenesis. Adv Exp Med Biol 2008; 636: 212-33.

28 Setchell B. Blood-testis barrier. In: Knobil E, Neill J, editors. Encyclopedia of Reproduction. New York: Academic Press; 1998. pp375-81.

29 Fawcett D. Ultrastructure and function of the Sertoli cell. In: Hamilton D, Greep R, editors. Handbook of Physiology. Washington, DC: American Physiological Society; 1975. p21-5.

30 Dym M, Fawcett DW. The blood-testis barrier in the rat and the physiological compartmentation of the seminiferous epithelium. Biol Reprod 1970; 3: 308-26.

31 Fawcett DW, Leak LV, Heidger PM. Electron microscopic observations on the structural components of the blood-testis barrier. J Reprod Fertil 1970; (Suppl 10): 105-22.

32 Dym M. The fine structure of the monkey (Macaca) Sertoli cell and its role in maintaining the blood-testis barrier. Anat Rec 1973; 175: 639-56.

33 Qian X, Mruk DD, Wong EW, Cheng CY. Breast cancer resistance protein regulates apical ectoplasmic specialization dynamics stage-specifically in the rat testis. Am J Physiol Endocrinol Metab 2013; 304: E757-69.

$34 \mathrm{Su}$ L, Mruk DD, Cheng CY. Drug transporters, the blood-testis barrier and spermatogenesis. J Endocrinol 2011; 208: 207-23.
35 Mruk DD, Su L, Cheng CY. Emerging role for drug transporters at the blood-testis barrier. Trends Pharmacol Sci 2011; 32: 99-106.

36 Planas JM, Alfaras I, Colom H, Juan ME. The bioavailability and distribution of transresveratrol are constrained by ABC transporters. Arch Biochem Biophys 2012; 527: $67-73$

37 Meyer ZU Schwabedissen HE, Kroemer HK. In vitro and in vivo evidence for the importance of breast cancer resistance protein transporters (BCRP/MXR/ABCP) ABCG2). Handb Exp Pharmacol 2011; 201: 325-71.

38 Takada T, Suzuki H, Gotoh Y, Sugiyama Y. Regulation of the cell surface expression of human BCRP/ABCG2 by the phosphorylation state of Akt in polarized cells. Drug Metab Disposit 2005; 33: 905-9.

39 Xie Y, Xu K, Linn DE, Yang XP, Guo Z et al. The 44-kDa Pim-1 kinase phosphorylates BCRP/ABCG2 and thereby promotes its multimerization and drug-resistant activity in human prostate cancer cells. J Biol Chem 2008; 283: 3349-56.

40 Enokizono J, Kusuhara $\mathrm{H}$, Ose A, Schinkel AH, Sugiyama Y. Quantitative investigation of the role of breast cancer resistance protein (Bcrp/Abcg2) in limiting brain and testis penetration of xenobiotic compounds. Drug Metab Disposit 2008; 36: 995-1002.

41 Hartz AM, Mahringer A, Miller DS, Bauer B. 17 -Estradiol: a powerful modulator of blood-brain barrier BCRP activity. J Cerebral Blood Flow Metab 2010; 30: 1742-55.

42 Zhang Y, Zhou G, Wang H, Zhang X, Wei F et al. Transcriptional upregulation of breast cancer resistance protein by $17 \beta$-estradiol in ER $\alpha$-positive MCF-7 breast cancer cells. Oncology 2006; 71: 446-55.

43 Wang RS, Yeh S, Tzeng CR, Chang C. Androgen receptor roles in spermatogenesis and fertility: lessons from testicular cell-specific androgen receptor knockout mice. Endocr Rev 2009; 30: 119-32.

44 Carreau S, Hess RA. Oestrogens and spermatogenesis. Philos Trans R Soc Lond B Biol Sci 2010; 365: 1517-35

45 O'Donnell L, Robertson KM, Jones ME, Simpson ER. Estrogen and spermatogenesis. Endocr Rev 2001; 22: 289-318.

46 Cheng CY, Mruk DD. A local autocrine axis in the testes that regulates spermatogenesis. Nat Rev Endocrinol 2010; 6: 380-95.

47 Jonker JW, Buitelaar M, Wagenaar E, van der Valk MA, Scheffer GL et al. The breast cancer resistance protein protects against a major chlorophyll-derived dietary phototoxin and protoporphyria. Proc Natl Acad Sci USA 2002; 99: 15649-54.

48 Enokizono J, Kusuhara H, Sugiyama Y. Effect of breast cancer resistance protein (Bcrp/ Abcg2) on the disposition of phytoestrogens. Mol Pharmacol 2007; 72: 967-75.

49 Robaire B. Advancing towards a male contraceptive: a novel approach from an unexpected direction. Trends Pharmacol Sci 2003; 24: 326-8.

50 Melaine N, Lienard MO, Dorval I, Le Goascogne C, Lejeune H et al. Multidrug resistance genes and $\mathrm{P}$-glycoprotein in the testis of the rat, mouse, guinea pig, and human. Biol Reprod 2002; 67: 1699-707.

51 Su L, Cheng CY, Mruk DD. Drug transporter, P-glycoprotein (MDR1), is an integrated component of the mammalian blood-testis barrier. Int J Biochem Cell Biol 2009; 41: 2578-87.

52 Flens MJ, Zaman GJ, van der Valk P, Izquierdo MA, Schroeijers AB et al. Tissue distribution of the multidrug resistance protein. Am J Pathol 1996; 148: 1237-47.

53 Wijnholds J, Scheffer GL, van der Valk M, van der Valk P, Beijnen JH et al. Multidrug resistance protein 1 protects the oropharyngeal mucosal layer and the testicular tubules against drug-induced damage. J Exp Med 1998; 188: 797808

54 Cheng CY, Mruk DD. Regulation of spermiogenesis, spermiation and blood-testis barrier dynamics: novel insights from studies on Eps8 and Arp3. Biochem J 2011; 435: 553-62.

55 Cheng CY, Mruk DD. Actin binding proteins and spermatogenesis. Some unexpected findings. Spermatogenesis 2011; 1: 99-104.

56 Vogl AW, Vaid KS, Guttman JA. The Sertoli cell cytoskeleton. Adv Exp Med Bio/2008; 636: 186-211.

57 Russell LD, Peterson RN. Sertoli cell junctions: morphological and functional correlates. Int Rev Cytol 1985; 94: 177-211.

58 Young JS, Vogl AW. Focal adhesion proteins zyxin and vinculin are co-distributed at tubulobulbar complexes. Spermatogenesis 2012; 2: 63-8.

59 Young JS, Guttman JA, Vaid KS, Vogl AW. Cortactin (CTTN), N-WASP (WASL), and clathrin (CLTC) are present at podosome-like tubulobulbar complexes in the rat testis. Biol Reprod 2009; 80: 153-61.

60 Young JS, Guttman JA, Vaid KS, Vogl AW. Tubulobulbar complexes are intercellular podosome-like structures that internalize intact intercellular junctions during epithelial remodeling events in the rat testis. Biol Reprod 2009; 80: 162-74.

61 Cheng CY, Mruk DD. An intracellular trafficking pathway in the seminiferous epithelium regulating spermatogenesis: a biochemical and molecular perspective. Crit Rev Biochem Mol Biol 2009; 44: 245-63.

62 Grandvuinet AS, Vestergaard HT, Rapin N, Steffansen B. Intestinal transporters for endogenic and pharmaceutical organic anions: the challenges of deriving in-vitro kinetic parameters for the prediction of clinically relevant drug-drug interactions. J Pharm Pharmacol 2012; 64: 1523-48.

63 Hua WJ, Hua WX, Fang JJ. The role of OATP1B1 and BCRP in pharmacokinetics and DDI of novel statins. Cardiovasc Ther 2011; 30: e234-41. 